\title{
PÚBLICO VISITANTE Y EMERGENTE DEL MUSEO DE ANTIOQUIA
}

\author{
Mónica del Valle Flórez* \\ Claudia Giraldo** \\ Sandra Zapata ***
}

\section{I}

Recibido: Noviembre 2 de 2015 - Aceptado: Noviembre 27 de 2015

\section{RESUMEN}

Los museos son unos de los espacios culturales más mediáticos del panorama social contemporáneo de allí su interés por comprender las necesidades, valores y expectativas de sus visitantes para pensar estrategia de marketing, comunicación e innovación.

El artículo presenta resultados del "Estudio de público visitante y no visitante de Museos en Antioquia" realizado por la Institución Universitaria Colegio Mayor de Antioquia y el Politécnico Colombiano Jaime Isaza Cadavid. Se encuentra que emerge como nuevo perfil de visitante del museo la empleada doméstica y el jubilado; disminuye la asistencia del público experto así como de los de estratos 4,5 y 6 . El público extranjeros tienen alto nivel educativo, prefieren una experiencia museística cognitiva, introspectiva o artística, el nacional opta por la experiencia social, prefiere espacios en los que pueda conversar a aquellos en los que se requiera silencio, así como experiencias de ocio que permitan la relajación y el entretenimiento.

A los museos se les presentan oportunidades y retos que les implica cambios sustanciales de su concepción clásica.

Palabras clave: Estudio de públicos, museos, turismo cultural, Medellín, consumo cultural, educación en museos, estudios culturales.

*Mónica María Valle F. Doctora en Estudios Científico Sociales del Instituto Tecnológico de Occidente - ITESO (México). Magister en Comunicación de la Universidad Iberoamericana-UIA (México), Especialista en Gerencia de la Comunicación de la Universidad Pontifica Bolivariana (Colombia) y Comunicadora Social - Periodista de la Universidad de Antioquia (Colombia). Docente e investigadora - Politécnico Colombiano Jaime Isaza Cadavid. Email:mmvalle@elpoli.edu.co

${ }^{*}$ Claudia María Giraldo Velásquez. Matemática y Especialista en estadística de la Universidad Nacionalsede Medellín. Magister en educación de la Pontificia Universidad Javeriana. Doctora en turismo de la Universidad de San Martin de Porres- Lima-Perú. Directora del Grupo de Investigación Empresa y Turismo- GIET. Docente e investigadora de la Institución Universitaria Colegio Mayor de Antioquia. Email:cgiraldo@colmayor.edu.co

*** Doctora en Gestión de Infraestructuras Aeronáuticas, Universidad de Enna, Italia. Profesora Asociada y Directora de la Unidad de Investigación del Departamento de Administración en la Facultad de Ciencias Económicas y Administración de la Universidad de la República, Uruguay. Sus temas de interés en investigación incluyen turismo y crecimiento económico, turismo cultural (museos), la industria de los cruceros y sus impactos en los destinos, así como temas de gestión aeroportuaria. Email: szapata@ccee.edu.uy 


\title{
EMERGING AUDIENCE AND VISITORS TO THE MUSEUM OF ANTIOQUIA
}

\begin{abstract}
SUMMARY
Museums are ones of the most media communicative cultural spaces of the contemporary social panorama, hence their interest in understanding the needs, values and expectations of their visitors, in order to carry out strategies about marketing, communication and innovation.

This paper introduces the findings of "Study of regular and non-regular visitors of museums in Antioquia", conducted by Institución Universitaria Colegio Mayor de Antioquia and Politécnico Colombiano Jaime Isaza Cadavid. It was found that a new visitor profiled has emerged, such as housekeepers and retired men; on the contrary, the art experts' attendance and the population ranked in 4, 5, and 6 socio economic strata have decreased. In regards to the foreign audiences, as they have a high level of education, they prefer a cognitive, introspective or artistic museum experience, in contrast to the local audience that prefer a social experience, attending sites where conversation, relaxation and leisure activities take place, instead of those in which silence is required.
\end{abstract}

Keywords: Public studies, museums, cultural tourism, Medellin, cultural consumption, training on museums, cultural studies.

\section{PÚBLICO VISITANTE E EMERGENTE DO MUSEU DE ANTIOQUIA}

\section{RESUMO}

Os museus são alguns dos espaços mediáticos mais cultural do panorama o contemporâneo social de lá o interesse deles/delas entender as necessidades, valores e as expectativas das visitas deles/delas para pensar estratégia de marketing, comunicação e inovação.

O artigo apresenta os resultados que correspondem ao perfil do público visitante e emergente do Museu de Antioquia, documento de síntese no qual figuram alguns dos trabalhos do Mestre Fernando Botero. Aparecem a empregada doméstica e o aposentado como novo perfil de visitantes; diminui a freqüência do público especialista, como também os estratos 4,5 e 6.

Enquanto o público estrangeiro prefere um experiência museística cognitiva introspectiva ou artística, o nacional opta pela experiência social, ele prefere esses espaços nos que eles podam bater-papo a esses onde é requerido silêncio como também experiências de lazer que permitam o relaxamento e o entretenimento. A este público o aborrece o silêncio, querem falar con a sua família e amigos, prefere a interação social mais do que a contemplação, a diversão mais do que o conhecimento.

Aos museus se lhes apresentam oportunidades e desafios que os insinua a mudanças significativas da sua concepção clássica.

Palavras chaves: Estudo de públicos, museus, turismo cultural, Medellín, consumo cultural, educação em museus, estudos culturais. 


\section{INTRODUCCIÓN}

a misión de los museos está cambiando de manera significativa, tradicionalmente se han dirigido, a la adquisición, conservación, investigación y exposición de objetos, ahora se han convertido en entidades de conocimiento y ocio (Capriotti, 2013). Para Hood (1983) es preciso mostrar el museo como un lugar donde se puede interactuar en familia, disfrutar y relajarse con los amigos, no solamente como un lugar en el que se aprende o se encuentran retos.

Los museos en Antioquia tienen un papel cada vez más significativo como centro dinamizador de la cultura y como entidades de servicio para el ciudadano local y para el turista. La capacidad de los museos para identificar y desarrollar sus potencialidades es un buen fundamento para la exitosa consecución de sus objetivos [1].

En Colombia, la política nacional y de turismo cultural reconocen como elemento esencial en la gestión de estos espacios, el conocimiento preciso de su demanda a través de investigaciones que permitan orientar sus estrategias de gestión territorial y acciones promocionales (ECD, 2010). Se sabe que los colombianos asisten poco a los museos, por debajo de la media nacional se encuentra la región central, de la cual hace parte Antioquia. En esa región, los estudios de publico, se lideran desde la Facultad de Arte de la Universidad de Antioquia; los de comunicación en museos desde las Facultades de comunicación. Se sugieren análisis interdisciplinarios (Valle, 2014;73)

En este panorama es necesario que los museos "desarrollen la audiencia" [2], lo que en palabras de Walts (2006) implica conocer mejor las necesidades de los visitantes. Margaret Hart Robertson, coordinadora de la ULPGC del Proyecto de la Universidad Metropolitana de Londres, implica que es necesario que el museo se transforme en espacio 'vivo' para nuevas formas de ver, en lugar de encuentro para nuevas comunidades, en vez de espacio 'muerto', protector del pasado.

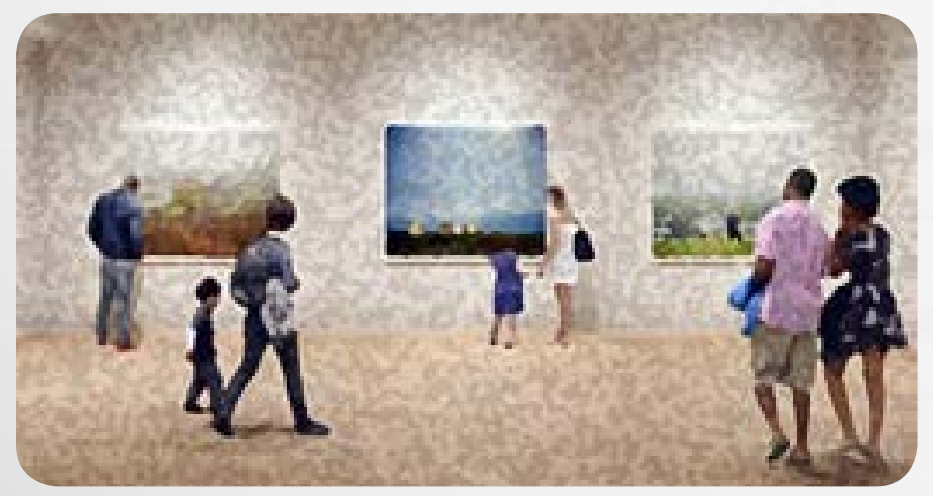

El Museo de Antioquia, la entidad para la que se realizó el estudio, se creó en 1881; en 1997 fue trasladado a la Plaza Botero, sector de la Veracruz en el centro de Medellín, convirtiéndose en eje central del proceso de transformación de dicha zona [3], tiene por misión:

Coleccionar, conservar, interpretar y promover el patrimonio de Antioquia, con el fin de dinamizar las prácticas artísticas y las culturas para dignificar al ser humano y contribuir a la transformación social, a través de una interacción educadora con la comunidad local, nacional e internacional [4].

El recinto cuenta con la exposición permanente de arte antioqueño de los siglos XIX y XX, arte internacional, con una exhibición de los pueblos originarios de América, culturas indígenas, africanas y europeas, así como con la más representativa obra del Maestro Fernando Botero.
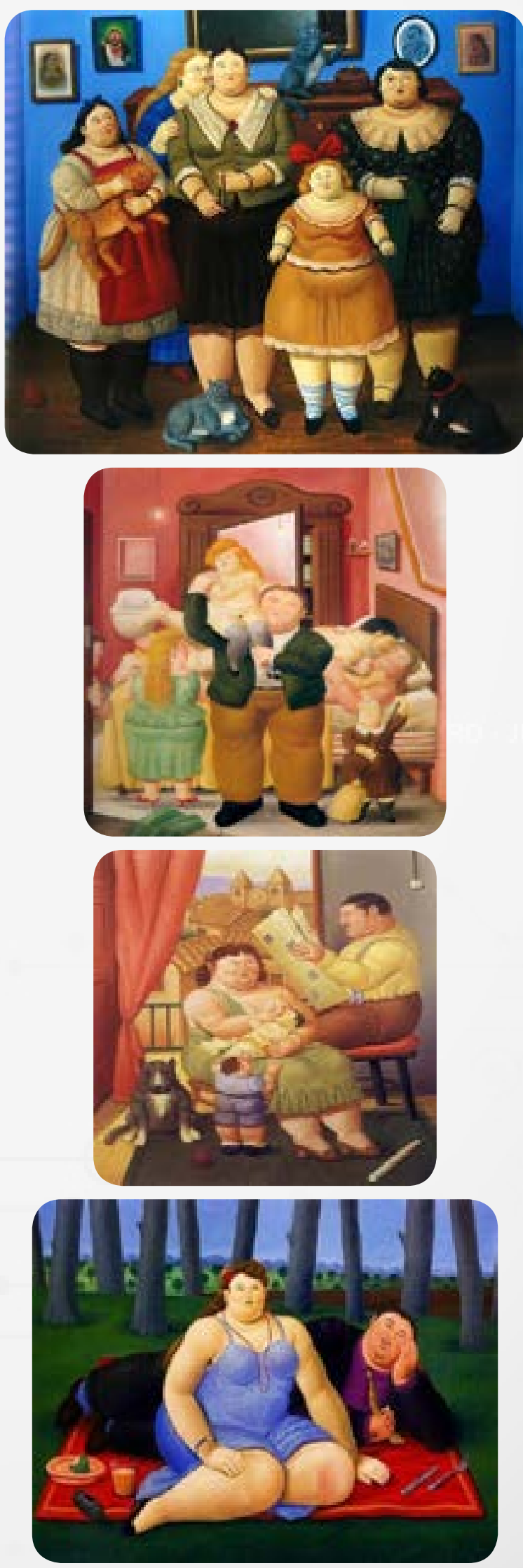


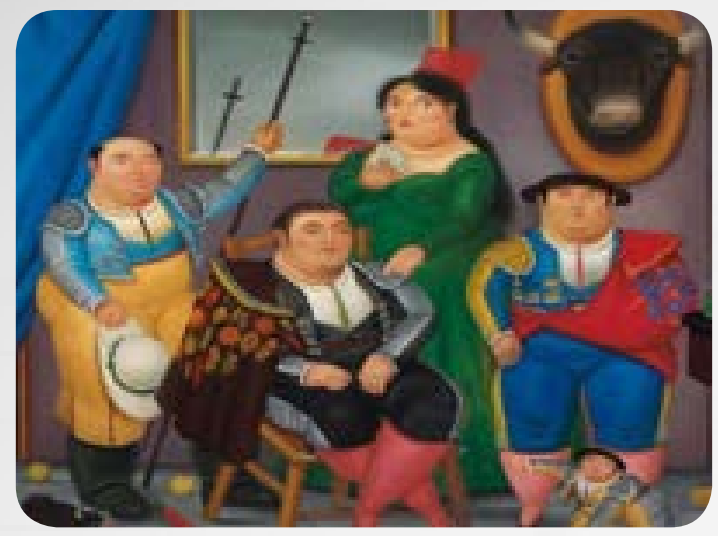

Este museo se ubica en la categoría de museo tradicional [5] o clásico [6]. Tiene por visión:

"la producción de conocimiento sobre las prácticas artísticas, los patrimonios y las culturas como factores que contribuyen a la transformación social" [7].
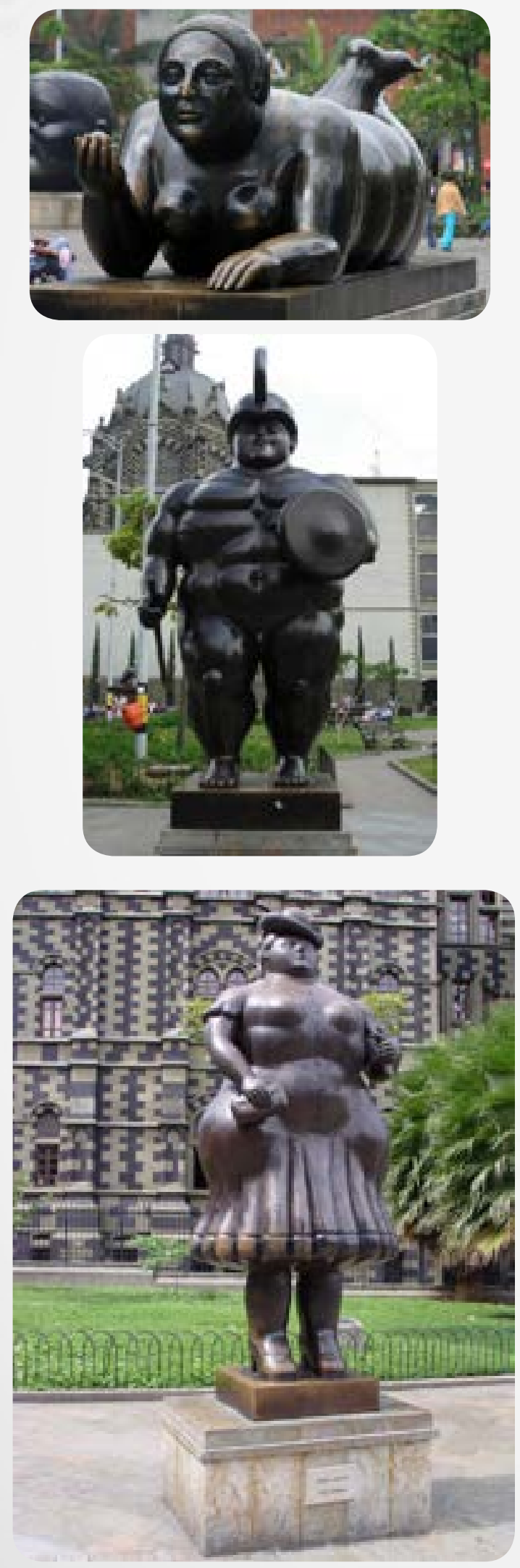

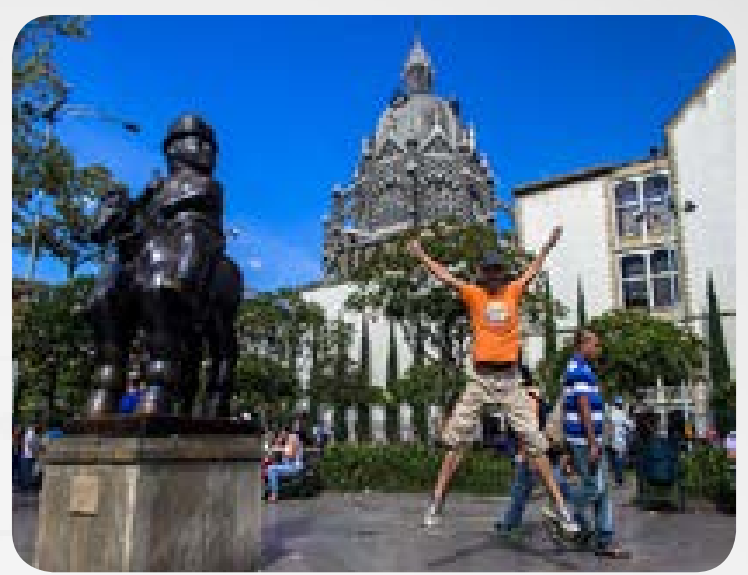

El estudio que se presenta tuvo entre sus objetivos determinar el perfil de los visitantes de un museo de la región antioqueña de Colombia; su lugar de procedencia, conocer los medios por los que se entera de las acciones del museo, el uso del tiempo libre, entre otros aspectos.

\section{METODOLOGIA}

El estudio tuvo un alcance descriptivo y correlacional, utilizó metodología cualitativa y cuantitativa. Se aplicó encuesta y se realizaron tres (3) grupos focal. El análisis se realizó en el programa estadístico informático SPSS. Un ejemplo de este se puede observar en la siguiente tabla en la que se presenta la sistematizan de los datos obtenidos a la pregunta por las actividades que realizaban los encuestados en su tiempo libre.

\section{Tabla N1.}

\begin{tabular}{|c|c|c|c|c|c|c|c|c|}
\hline \multirow{2}{*}{ versosurs } & \multirow[t]{2}{*}{ " } & \multirow[t]{2}{*}{ E.T } & \multirow[t]{2}{*}{ wois } & \multirow[t]{2}{*}{ t } & \multirow[t]{2}{*}{147} & \multirow[t]{2}{*}{ topial } & \multicolumn{2}{|c|}{ AC sw pare $200(1)$} \\
\hline & & & & & & & nomen & Supene \\
\hline 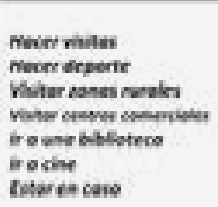 & 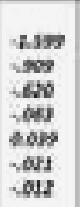 & 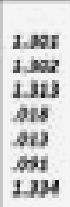 & 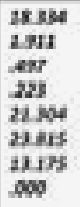 & $\begin{array}{l}4 \\
i \\
i \\
i \\
1 \\
i \\
i \\
i\end{array}$ & 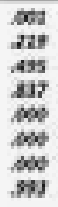 & 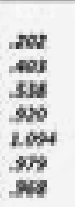 & 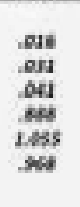 & 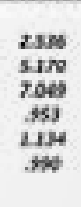 \\
\hline
\end{tabular}

La población objeto de estudio estuvo conformada por: visitantes del Museo de Antioquia en los meses de julio y agosto de 2014 (400 visitantes), estudiantes de colegios de Medellín de estratos 1, 2, 3, 4, 5 , 6, (400 estudiantes), visitantes de centros comerciales (100visitantes): Monterrey y Unicentro, expertos y administrativos docentes y empleados del Politécnico Colombiano Jaime Isaza Cadavid y de la Institución Universitaria Colegio Mayor de Antioquia (15 de cada institución).

Algunas de las preguntas en los grupos focales fueron: ¿qué hace en su tiempo libre', ¿ ha visitado el Museo de Antioquia o algún otro museo?, ¿Qué concepto tiene de lo que es cultura?, ¿ tiene sentido hablar 
de cultura de élite y cultura popular?, ¿la visita a un museo requiere capacidades intelectuales?, ¿por qué las personas no van a los museos?, si ha visitado museos fuera del país ¿cuál ha sido su experiencia? ¿qué lo motiva visitar un museo?, ¿qué tendría que hacer el museo de Antioquia para que el público lo quisiera visitar?.

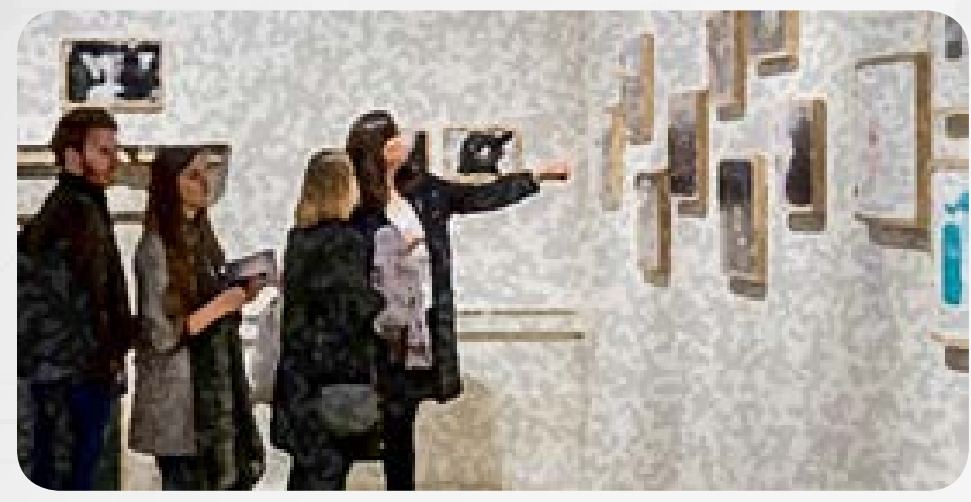

\section{RESULTADOS}

Después de la sistematización de datos y su interpretación se determinan los siguientes perfiles de público visitante del museo:

\subsection{Público local}

Perfiles 1: Estudiante. Mujeres y hombres, entre 18 y 34 años, con nivel educativo de secundaria y universitario [8]. Los de secundaria se ubican en los estratos 1, 2 y 3 . A diferencia de los universitarios que pertenecen a estratos 4,5 y 6 . Hace también parte de este perfil los niños y niñas de primaria de estratos 1 , 2 y 3 . Perfil 2. Mujeres y hombres entre 35 y los 54 años, técnicos y tecnólogos, de estratos 1,2 y 3 .
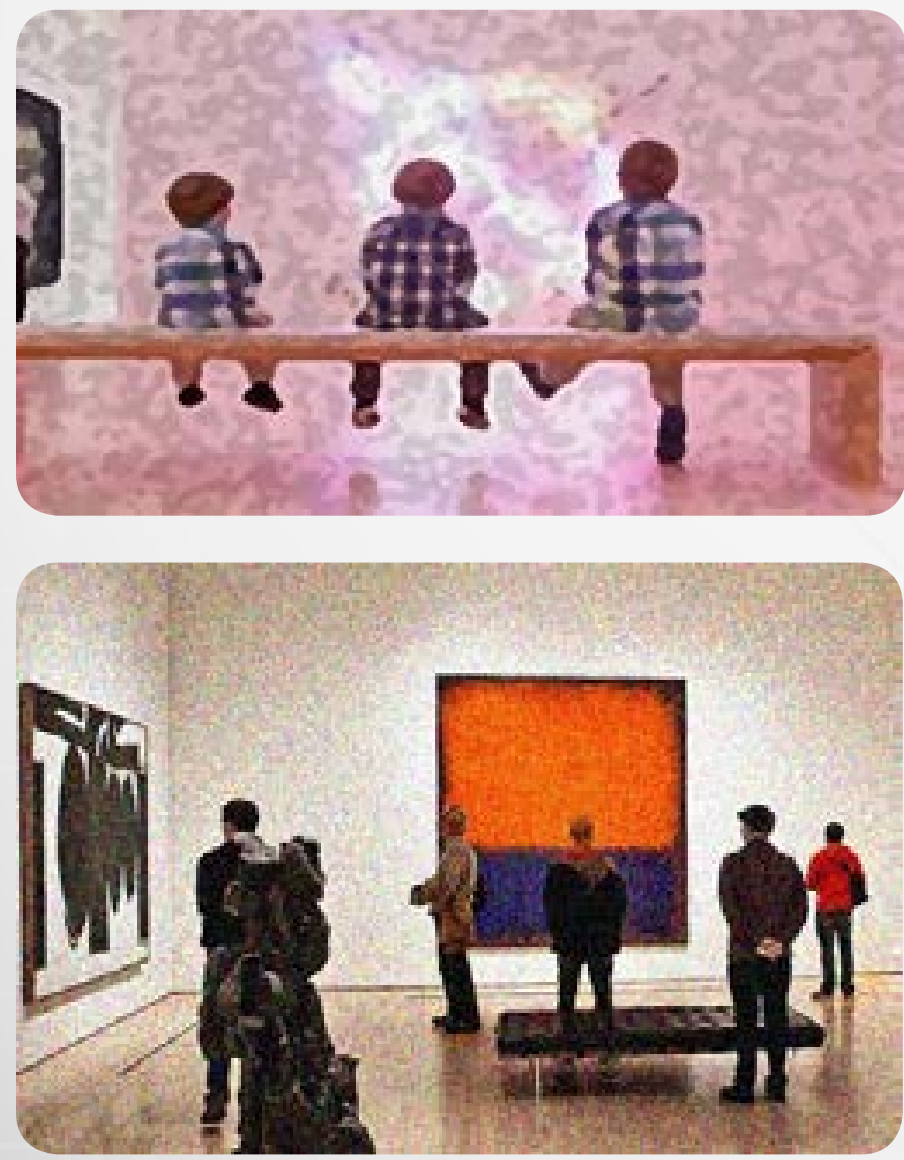

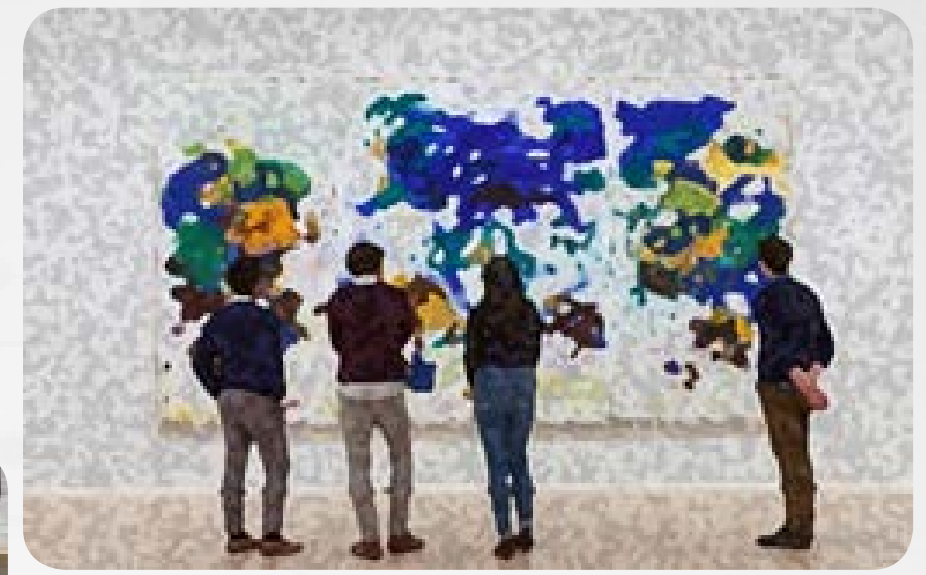

La presencia del estrato 1, 2 y 3, a nivel de estudiantes y personal en general, quizás se deba a los apoyos estatales para visita de públicos a museos, estos consisten en que pueden ingresar de manera gratuita, con solo presentar la factura de servicios públicos, cancelada. Es de aclarar que el museo para el que se efectúo el estudio es de carácter privado y tienen un costo por el ingreso al recinto.

Público local emergente: en cuanto a ocupaciones emergentes, en términos de visitantes, está el de empleadas domésticas y la de los jubilados. Público local potencial. Estudiantes, ejecutivos y profesionales de estratos 4, 5 y 6 . Artistas, profesores y personal del sector de la cultura. Se encuentra que el $66 \%$ de quienes visitan el Museo son solteros.

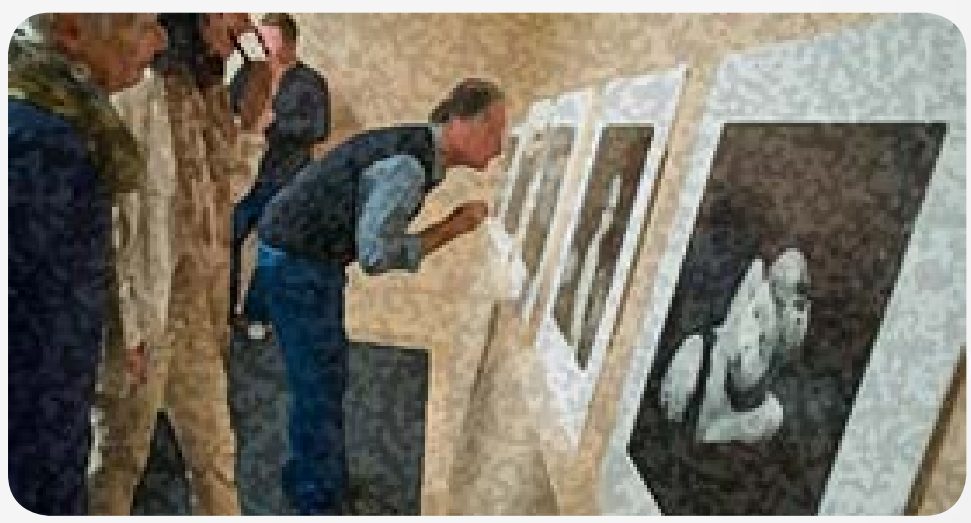

\subsection{Perfil público nacional}

Perfil 1: Hombres y mujeres de la ciudad capital del país - Bogotá. Asisten al museo por curiosidad, porque es famoso, para conocer las obras expuestas. Generalmente están en la región por vacaciones o por trabajo. Tienen entre 18 y 34 años. 
Grafica N1.

Lugar de procedencia del visitante Nacional

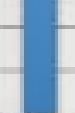

(1.

1.tris:

El $49 \%$ de los que visitan el museo son de Medellín, seguidos de los procedentes de Bogotá (12\%). Los visitantes del municipio de Bello, también presentan cierta frecuencia de visita (6\%), es de señalar que estos datos coinciden con los reportados en el informe 2013 [9], en el que se indica que el Museo es visitado principalmente por colombianos $(89 \%)$, de estos el $(50 \%)$ de Medellín y el $(14,25 \%)$ de Bogotá. El dato sugiere estrategias para atraer visitantes de otras zonas del país y especialmente de otros municipios de Antioquia, ya que como se indicó en el informe 2013, hay muy baja presencia de visitantes del área Metropolitana y de otras regiones del Departamento [10].

\subsubsection{Procedencia del Visitante de Medellín}

El $5 \%$ de los visitantes son del barrio Manrique, el $4 \%$ de Robledo, 3\% de Aranjuez, el $2 \%$ de Belén. Estos porcentajes evidencian la presencia de personas de estratos 1,2 y 3 en el museo.

Grafica N2.

Lugar de prosedencia del visitante de Medellín

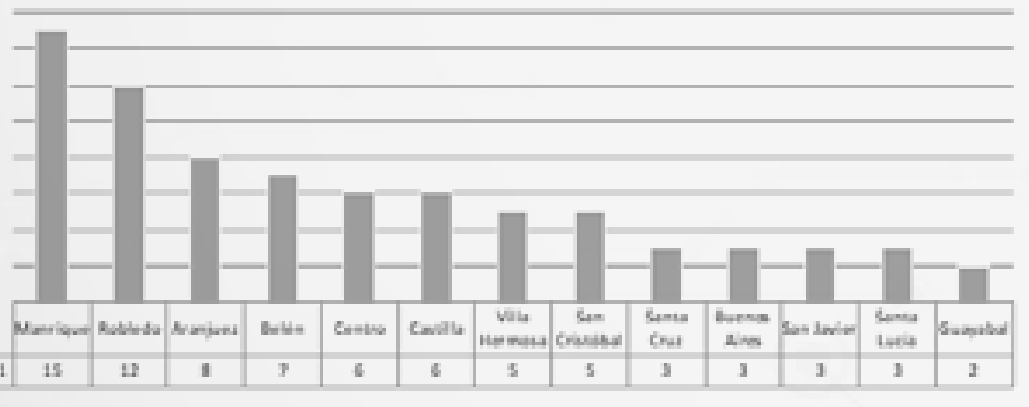

Los estudiantes que no visitan museos, en su tiempo libre prefieren practicar algún. En general los jóvenes de nivel 11 de todos los estratos, son los que menos visitan el museo. Prefieren tener actividades que conlleven interacción con diferentes personas. Manifiestan que aunque han sido motivados a visitar museos: los estratos 1,2 y 3 por la Institución educativa y las estratos 4, 5,6 por la familia. Los que le han visitado, lo han hecho en compañía de amigos. Un porcentaje significativo manifestó que no ha regresado al museo porque no les llama la atención.

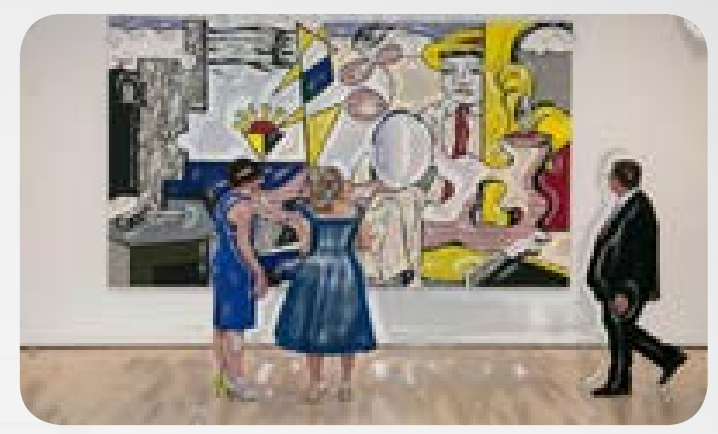

El $50 \%$ de los estudiantes de estrato 1,2 y 3 , no logran describir su experiencia al visitar museos; el otro $50 \%$ considera que dichos recintos son para gente con mucha formación, sitios estáticos, lugares para gente mayor, que en ellos es hay que estar "callados como en una iglesia o en una biblioteca"; indican también que estar en el museo es una experiencia "aburrida" y que son para gente de estratos altos. Los estudiantes de estratos 4,5 y 6 consideran que la experiencia de visitarle es un motivo para aprender, es interesante y enriquecedora, y que estos recintos son para todo tipo de público.

Los encuestados locales, que han tenido la oportunidad de visitar museos por fuera de Colombia, consideran que esos son mucho más dinámicos y creativos, que en ellos hay actividad interactiva, lo que hace más enriquecedora la experiencia.
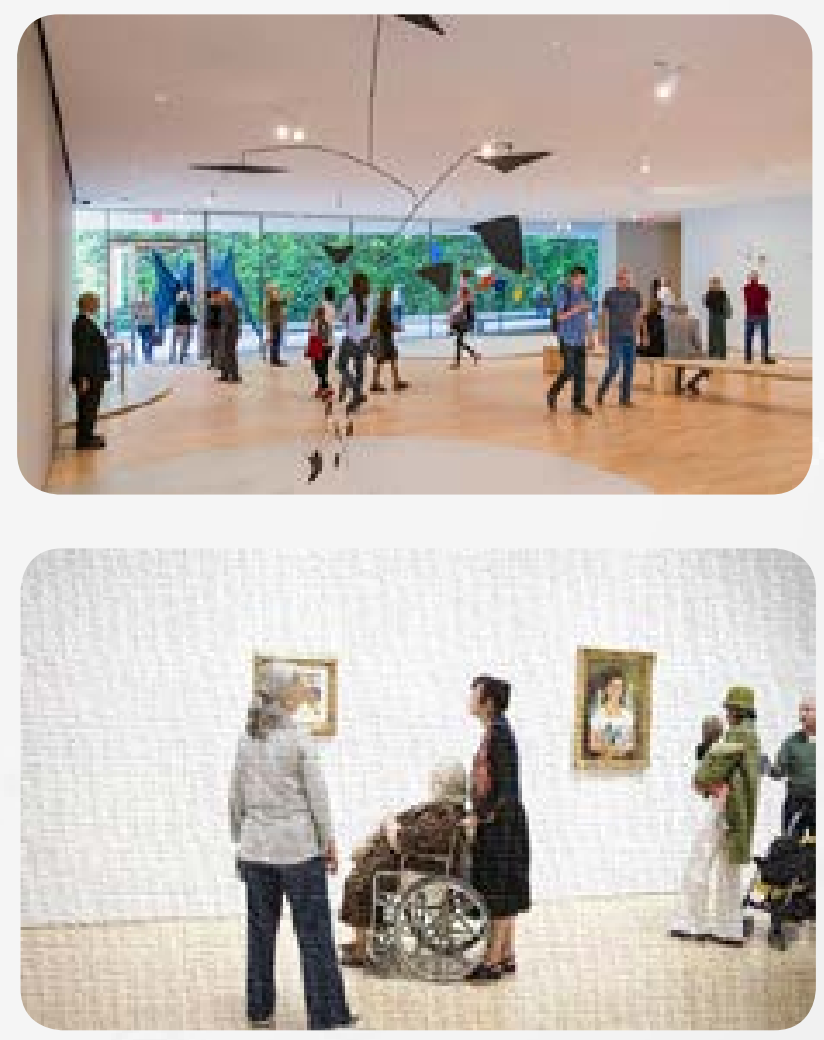

\subsection{Público Extranjero}

Perfil 1. Mujeres y hombres entre 18 y 34 años, con Grado Universitario, de Maestría o PhD, en su mayoría empleados. Estos visitantes en su mayoría son solteros, proceden de México, EE.UU y Canadá. Un alto porcentaje de estos es empleado o estudiante universitario. 


\section{Grafica N3}

Lugar de procedencla del vistante extranjero

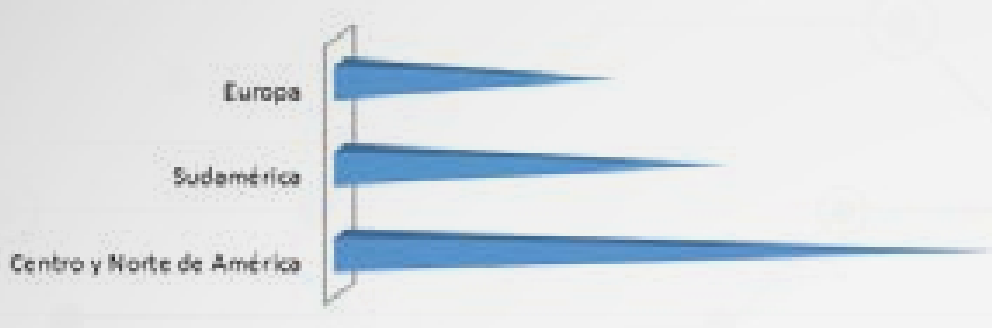

En comparación con el informe de 2013 [11], se encuentra un aumento en ese ítem, en tanto se amplió la visita de centro y norteamericanos, así como de suramericanos y europeos, es de señalar que los estadounidenses y los mexicanos son los visitantes más asiduos del Museo.

Público extranjero potencial. Suramericanos, extranjeros jubilados: Los mayores de 55 son quienes menos visitan el Museo, una hipótesis de esto podría ser que no es el tipo de extranjero que más visita Colombia. Se sabe que en Europa los mayores de 50 años son quienes más visitan museos. Otro público potencial son los extranjeros que viven en MedellínAntioquia-Colombia.
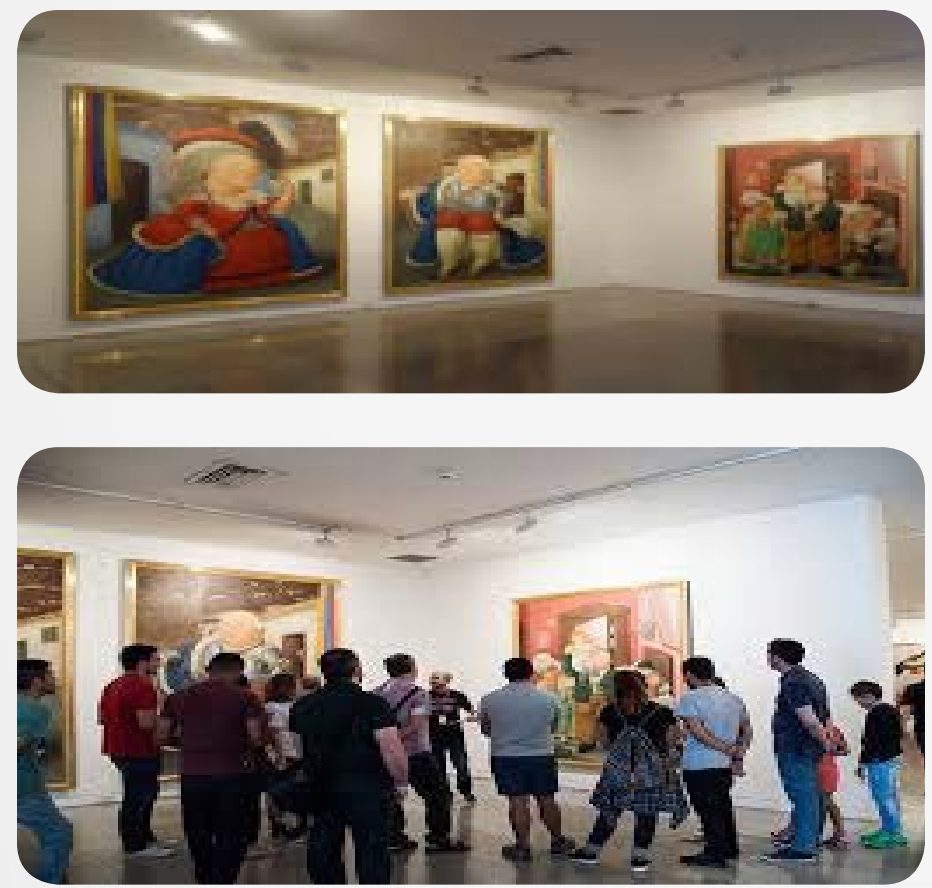

La mayoría de visitantes extranjeros que van al museo lo hacen por recomendación de amigos; son muy pocos los que indican que van por la información suministrada por los guías turísticos de la Ciudad, o por la guía /libro de turismo. Los encuestados manifiestan que se enteran de las actividades culturales por medio del email, las redes sociales y página Web del museo (12\%). Este público prefiere actividades de ocio, en las que tenga la oportunidad de aprender. Los nacionales prefieren la interacción social.

En la construcción del perfil psicográfico de los visitantes de museos, Kotler y Kotler (2001) \& Slater (2006) mencionan que las principales motivaciones a la hora de visitar museos son el escapismo, el aprendizaje y la interacción social [12]. Para Yellis (1985) las jerarquía de las 5 necesidades de Maslow: psicológicas, seguridad, social, autoestima y autoactualización, influyen en las decisiones relacionadas con el ocio. Para el público joven y con un alto nivel educativo, las visitas a las galerías de arte moderno son una experiencia de ocio, motivada por el deseo de relajación y de entretenimiento [13].

Otro tipo de perfil de visitantes del museo son los "asiduos" que representan el (57\%) y visitantes "nuevos" que llegan a representar el (43\%).

\section{Conclusiones}

El estudio y observación de públicos de los museos se constituye en una importante estrategia en el "desarrollo de la audiencia" expresión que alude a mejorar los servicios para los visitantes: múltiples experiencias, autodesarrollo, mejorar la comunicación con estos, el acceso, interacción y segmentación de públicos. Dinámica necesaria para que los museos logren competir en la industria del entretenimiento, el ocio y el tiempo libre, puedan llevar a cabo su misión, la cual se hace más compleja al encontrar pocos recursos económicos para la cultura, además de nuevos públicos con diversas expectativas y motivaciones en torno a estos recintos.

Y es que la motivación reverencial y educativa de la visita a los museos, con las que Falk y Dierking (1992) caracterizaron a los públicos del Reino Unido en los 70 y 80 , parece ya cosa del pasado, la recreación social, esto es la diversión y el entretenimiento en un ambiente seguro con familia y amigos, parece ser lo que prima al momento de elegir en que ocupar el tiempo libre. En el estudio realizado se encuentra que la curiosidad, también aparece como motivación que lleva al nuevo público al museo. Se configura un público al que le molesta el silencio, quiere hablar con su familia y amigos, prefiere la interacción social más que la contemplación, la diversión al conocimiento.

En este contexto el museo denominado clásico tiene las horas contadas. Cambia o lo cambian. ¿Hay que cambiar al museo o las expectativas del público?, cada museo se lo responderá; por lo pronto, en relación al estudio efectuado y del que se da cuenta en este artículo, se encuentra que otros públicos le visitan, lo que se constituye en reto y en una gran oportunidad para la cultura. Estudiantes, empleados y en general el estrato 1,2 y 3 de Medellín asisten más frecuentemente al museo, no tantas veces como las estadísticas quisieran, pero más que en épocas pasadas. Quizás esto se deba a una política de entrada gratuita con recibo de servicios públicos,cancelada. 
Resulta interesante para este estudio encontrar que las empleadas domésticas así como los jubilados se tornan público emergente del museo estudiado. Se deben generar estrategias de fidelización de público así como atraer nuevamente al museo al de estrato 4,5 y 6 , y al visitante experto, el que actualmente visita poco este recinto, estos presentan interés en una experiencia museística excepcional y de alto contenido cultural.

La audiencia por desarrollar en el museo, corresponde al público local y nacional, aunque para el recinto le resulte más ideal el extranjero, en tanto puede comprar boleta de ingreso a tarifa plena. Una buena noticia es que el museo no tienen género, tanto hombre como mujeres le visitan. Ello desde una clasificación clásica, ¿tendremos que preguntarnos por la comunidad LGTBI y grupos minoritarios que asisten al museo? $Y$ por supuesto ¿si hay que ir al museo o "sacar" el museo?

Se encuentra en el estudio que los solteros van al museo más que los casados, también asisten más los universitarios de estratos 4,5 y 6; ¿Qué pasa con los de estratos 1, 2 y 3 que asisten poco? La pregunta se lanzó en una clase con estudiantes universitarios de estos estratos, la respuesta, "yo prefiero irme al lleras [Zona rosa de Medellín] y tomarme una cerveza". Sería recomendable generar estrategias para que este tipo de público se acerque al museo.

En el estudio realizado se identifican públicos potenciales en cada perfil tales como los extranjeros Suramericanos, los ejecutivos y profesionales locales y los profesores de arte. También son potenciales las figuras del benefactor y del que podría hacer servicio social en estos recintos. Lo importante es determinar ¿qué públicos? ¿ por qué? y ¿para qué?.

El museo requiere estrategias para desarrollar público virtual. La página web y demás instrumentos del mundo digital deben ser aplicadas, introduciendo nuevas posibilidades y mejorando las existentes, ya que en la actualidad el público se aproxima al museo por medios electrónicos y también desea actividades que implique la utilización de estos.

Se concluye que el museo estudiado, cumple una función social y cultural importante en la región antioqueña, sus estrategias educativas, comunicativas y de gestión ha permitido ampliar el acceso a grupos sociales poco favorecidos como son los estratos 1 , 2 y 3 . Estas estrategias financiadas directamente por los entes gubernamentales de la región, no son suficientes para el sostenimiento de este recinto, por lo que debe desarrollar públicos, servicios, acciones en sí estrategias que le permitan ampliar el perfil de sus públicos y sus ingresos.

\section{BIBIOGRAFÍA}

Asensio \& Pol (2007). Conversaciones sobre el aprendizaje informal en museos y el patrimonio. En Turismo, Patrimonio y Educación, Fernández Betancort, editor. Escuela Universitaria de Turismo de Lanzarote Adscrita a la Universidad de Las Palmas de Gran Canaria. Este libro se puede descargar en español y en ingles en la siguiente dirección como un E-BOOK. Colección Pasos Edita "REVISTA DE TURISMO Y PATRIMONIO CULTURAL” http://www. pasosonline.org/

Babin and M. Griffin (1998) The nature of satisfaction: an updated examination and analysis. Journal of Business Research vol 41 n², 127-

Beard JG, Ragheb MG (1983). Measuring leisure motivation. Journal of Leisure Research vol $15, n^{\circ} 3$, 219-228.

Beeho, A., \& Prentice, R. (1995). Evaluating the Experiences and Benefits Gained by Tourists Visiting A Socio-Industrial Heritage Museum: An Application of AESB Grid Analysis to Blists Hill Open-Air Museum, The Ironbridge Gorge Museum, United Kingdom. Museum Management and Curatorship, vol 14, $n^{\circ} 3$, 229-251.

Capriotti, P. (2013). Maniging Strategic Communication in Museums. The case of Catalan museums. Communication \& Society/ Comunicación y Sociedad, Vol. 26, n. 3, pp. 98-116).

ECD. Ministerio de Educación, Cultura y Deporte (2010). Conociendo a todos los públicos. ¿Qué imágenes se asocian a los museos? España. http://www.gestorcultural.org/images/noticies/ noticia227172512.pdf

Fernández, B. (2013). 50 experiencias prácticas para la gestión y fidelización de público en museos. Asimétrica. Disponible en http://asimetrica.org/wpcontent/uploads/2013/06/Fidelizacion-Museos.pdf (acceso septiembre 23 de 2013)

Hood, M. (1981). Staying away, Why people choose not to visit museums? Museum News.

Moreno- Gil, S. y Brent, J. R. (2009). Understanding the Museum Image Formation Process. Journal of Travel Research, vol $47 n^{\circ} 4,480-493$.

Rausell, Köster (2005). Un museo ¿Qué es desde la perspectiva económica y desde la lógica de acción pública? Disponible en: http://www.uv.es/museos/ MATERIAL/Rausell.pdf

Rodríguez, Ángel (2004), “Comunicación para estimular la visita al museo", en: Museo, núm. 9, pp. 115- 124. En Alarcón, R. (2007). Sociología y estudios 
de público en los museos españoles. Revista Museo. $X$ Jornadas de Museología- España. Consutlado 13/08/2015. Disponible en http://www.apme.es/ revista/museo12_233.pdf

(2002), "Nueva sociedad, nuevos museos. El papel del marketing en los museos", en: Revista de Museología, núms. 24-25, pp. 25-38.

Schmilchuk, Graciela (2007). Venturas y desventuras de los estudios de públicos. En Cuicuilco, México, nueva época, vol $3, n^{\circ} 7$, p. 31-57.

Slater, A. (2006). Escaping to the gallery: understanding the motivations of visitors to galleries. International Journal of Nonprofit and Voluntary Sector Marketing. Published online 13 December 2006 in Wiley InterScience.

Pincus J. 2004. The consequences of unmet needs: the evolving role of motivation in consumer research. Journal of Consumer Behaviour 3(4):375-387.

Valle, F. Mónica \& Otros (2005). Comunicación Organizacional. Abordajes y Perspectivas de análisis. Centro Internacional de Estudios Superiores de Comunicación para América Latina, CIESPAL.

Valle, F. Mónica (2014). La investigación en comunicación en Antioquia Colombia. Revista Luciérnaga/ Comunicación, Año 6, N12. Facultad de Comunicación Audiovisual- Politécnico Colombiano Jaime Isaza Cadavid-PCJIC \& Facultad de Ciencias de la Comunicación - Universidad Autónoma de San Luis Potosí- UASLP. México. ISSN 2027- 1557. Págs. 73-91. Disponible en: http://www.politecnicojic.edu.co/ images/downloads/publicaciones/revista-luciernaga/ luciernaga-12/pdf/5-investigacion_comunicacion_ espanol.pdf

Waltl, Christian (2006). Museums for visitors: Audience development - A crucial role for successful museum management strategies. INTERCOM. Conference Paper. Consultado el 11/08/2015. Disponible en http:// www.intercom.museum/documents/1-4waltl.pdf

\section{Notas}

[1] Este artículo es producto del "Estudio de público visitante y no visitante del Museo de Antioquia", financiado por la Institución Universitaria Colegio Mayor de Antioquia y el Politécnico Colombiano Jaime Isaza Cadavid.

[2] Esto es perfeccionar y mejorar la comunicación con los visitantes, lograr una audiencia que sea frecuente, convertir los no visitantes en visitantes, a los visitantes en visitantes frecuentes y a los asistentes regulares del museo en seguidores; mejorar el acceso, ofrecer múltiples experiencias, involucrar a los visitantes (manos y mentes), establecer una red activa con especiales grupos de destinatarios.

[3] Este museo cuenta con una exposición permanente distribuida alrededor de temas como la historia del arte antioqueño durante los siglos XIX y XX, las obras donadas por el Maestro Fernando Botero, arte internacional, los pueblos originarios de América, las culturas indígenas, africanas y europeas. Disponible en https://www.museodeantioquia.co/elmuseo/\#/mision-vision-y-objetivos/

[4] Misión Museo de Antioquia. Disponible en https:// www.museodeantioquia.co/el-museo/\#/mision-visiony-objetivos/

[5] “...Lugares concebidos como instituciones didácticas en las que se vuelve explícito un discurso pedagógico que inviste de valores prácticos toda la actividad expositiva". Estos valores pueden ser de corte nacional, reafirmado a través del arte producido históricamente por una comunidad humana... Zunzunegui, S. (2003:84).

[6] Presentan cierta monumentalidad, diseñados para la contemplación. Zunzunegui (2003:90).

[7] Colecciones Museo de Antioquia. Disponible en https://www.museodeantioquia.co/colecciones/

[8] Este dato resulta positivo en tanto, en el 2013, se encontraba que un alto porcentaje de Universitarios de Medellín, no visitaban el Museo). Informe "Análisis de Público Universitario para el Museo de Antioquia" (2013). Dra. Mónica Valle Flórez- Politécnico Colombiano Jaime Isaza Cadavid. Dra. Claudia Giraldo Velásquez . Dra. Sandra Zapata Aguirre. Institución Universitaria Colegio Mayor de Antioquia.

[9], [10] Informe "Análisis de Público Universitario para el Museo de Antioquia” (2013). Dra. Mónica Valle Flórez- Politécnico Colombiano Jaime Isaza Cadavid. Dra. Claudia Giraldo Velásquez. Dra. Sandra Zapata Aguirre. Institución Universitaria Colegio Mayor de Antioquia.

[11] En el Informe "Análisis de Público Universitario para el Museo de Antioquia" (2013), se encontró que el $(89 \%)$ de visitante eran colombianos, el $(2 \%)$ provenían de Estados Unidos de Norte América, el (1\%) venían de Venezuela, Ecuador y España.

[12] Slater, A. (2006). Escaping to the gallery: understanding the motivations of visitors to galleries. International Journal of Nonprofit and Voluntary Sector Marketing.

[13] Proyecto de Investigación Turismo Cultural, segmentando a los turistas que van en busca de la realización de un turismo cultural. Estudio adelantado por Richards en Europa para la Asociación para el Turismo y Educación de Ocio (ATLAS). 
Para citar este artículo:

Valle, F. Mónica; Giraldo, V. Claudia y Zapata, A. Sandra (2017). Público visitante y emergente del Museo de Antioquia. Revista Luciérnaga/

Comunicación, Año 9, N17. Facultad de

Comunicación Audiovisual- Politécnico Colombiano

Jaime Isaza Cadavid-PCJIC \& Facultad de Ciencias

de la Comunicación - Universidad Autónoma de

San Luis Potosí- UASLP. México. ISSN 2027- 1557.

Págs. 38-47.

DOI. 10.33571/revistaluciernaga.v9n17a5

OJS. http://revistas.elpoli.edu.co/index.php/luc/issue/ archive

Link. http://www.politecnicojic.edu.co/index.php/ revista-luciernaga

\section{口象细 \\ (x) \\ (1)

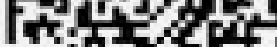

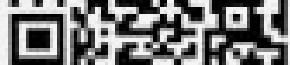 \\ orcid erg/0000-0003-1803-1115}

